\title{
Wake Up and Smell the Coffee: Yet Another No Go for Cardiac Patients?
}

\author{
Editorial to "Caffeinated Coffee Blunts the Myocardial Protective Effects of Statins \\ Against Ischemia-reperfusion Injury in the Rat" by Ye et al.
}

\author{
Niels P. Riksen • Derek J. Hausenloy • Derek M. Yellon
}

Published online: 16 April 2008

(C) The Author(s) 2008

As caffeine is one of the most widely consumed pharmacologically active compounds in the world, its possible association with cardiovascular disease has been the subject of scientific study for decades. Disappointingly, results from epidemiological studies on coffee intake, the most important dietary source of caffeine, have been equivocal. Whereas most case-control studies have reported an increased risk for cardiovascular disease in heavy coffee consumers, prospective studies have in general not supported such an association [1]. Only recently have preclinical studies begun to address the effect of caffeine on therapeutic infarct size-limitation. Brief periods of ischaemia have been shown to limit myocardial infarct size induced by a subsequent more prolonged ischaemic insult, a phenomenon which has been termed ischaemic preconditioning [2]. It transpired that various drugs, including the 3-hydroxy-3-methylglutaryl-coenzyme A (HMG-CoA) reductase inhibitors (statins), can mimic the infarct sizelimiting effect of ischaemic preconditioning. Furthermore, they confer cardioprotection when administered at the time of myocardial reperfusion [3]. As adenosine is a pivotal mediator of ischaemic preconditioning, we have recently studied the effect of caffeine, which is a non-selective adenosine receptor antagonist, in two experimental models of preconditioning in humans, and showed that the

N. P. Riksen $(\bowtie)$

Department of Pharmacology-Toxicology 149,

Radboud University Nijmegen Medical Centre,

P.O. Box 9101, 6500 HB Nijmegen, The Netherlands

e-mail: N.Riksen@aig.umcn.nl

N. P. Riksen • D. J. Hausenloy • D. M. Yellon

Hatter Cardiovascular Institute, University College London,

67 Chenies Mews,

London WC1E 6HX, UK protective effect of ischaemic preconditioning was completely abolished by pretreatment with caffeine [4].

Although the mechanism of statin-induced cardioprotection is less extensively studied than that of ischaemic preconditioning, there is evidence that this protection is also dependent on adenosine receptor stimulation: in dogs, administration of a statin immediately before coronary occlusion increased activity of ecto-5'-nucleotidase, the extracellular enzyme that catalyzes the conversion of adenosine monophosphate into adenosine, and reduced infarct size, which was abolished by pretreatment with an inhibitor of ecto-5'-nucleotidase or the adenosine receptor antagonist 8-sulfophenyltheophylline [5]. More recently, it has been reported that the adenosine uptake blocker dipyridamole could potentiate atorvastatin-induced cardioprotection, which was abolished by the adenosine receptor antagonist aminophylline [6]. Therefore, it is logical to assume that coffee consumption could adversely affect the cardioprotective properties of statins.

In this issue of the Journal, Ye et al. have studied this hypothesis in a rat in vivo model of myocardial infarction. In this elegant study, they showed that a 3-day treatment with atorvastatin reduced infarct size, which was completely abolished by concomitant administration of caffeinated coffee, but not decaffeinated coffee, in the drinking bottle [7]. The observation that decaffeinated coffee did not affect atorvastatin-induced protection suggests that caffeine is the compound responsible for the observed effects. Importantly, the plasma caffeine concentration in the caffeinated coffee-treated group averaged $6 \mathrm{mg} / \mathrm{l}$, which is in the same range as the concentration reached after drinking two cups of regular coffee [8]. Although the atorvastatin dose in this study $(10 \mathrm{mg} / \mathrm{kg})$ was much higher than the maximum dose given to humans $(80 \mathrm{mg}$ ), and although a pharmacokinetic interaction between caffeine and atorvastatin cannot be 
excluded, the findings of this study are of potential clinical importance.

Given that caffeine, which is abundantly present in significant quantities in coffee, tea, chocolate, and soft drinks, has been shown to abolish the cardioprotective effect of ischaemic preconditioning and statins, the question remains as to why previous epidemiological studies did not show a consistent association between coffee intake and cardiovascular disease? There are several possible explanations for this apparent discrepancy. It could be that the infarct size-limiting effects of ischaemic preconditioning and statin treatment are not of significant importance in the general population. However, there is some evidence that patients who have experienced brief periods of chest pain before an acute myocardial infarction, demonstrate a smaller infarct size [2]. In addition, there is evidence that the administration of statins can reduce myocardial damage in patients undergoing percutaneous coronary intervention because of chronic stable angina or an acute myocardial infarction $[9,10]$.

As these potential beneficial effects involve selected patient groups, it could be that any detrimental effect of coffee intake is not detected in large epidemiological trials in non-selected patients. The recent finding that coffee consumption is only associated with an increased cardiovascular risk in patients with a CYP1A2 genotype that predicts slow caffeine metabolism, emphasizes the possibility that coffee intake may have adverse effects in certain subgroups of patients [11]. The inhibition of ischaemic preconditioning will not increase the incidence in cardiovascular events per se, but rather would be expected to adversely affect outcome after an acute myocardial infarction in the subset of patients experiencing pre-infarction angina, a group of patients which in general have not been the subject of study in previous epidemiological trials. Moreover, in these studies, the average daily coffee intake is taken into account, and not the plasma caffeine concentration at the moment of the myocardial infarction, which is necessary to study its effect on preconditioning- or statin-induced protection. Most prospective epidemiological trials investigating the effect of coffee intake on cardiovascular morbidity and mortality were started before the general introduction of statin use for the primary and secondary prevention of cardiovascular disease [12]. Furthermore, it should be noted that the observed beneficial effect of atorvastatin by Ye et al. probably involves a non-lipid lowering ("pleiotropic") effect, although in this study plasma lipid levels were not measured, and that coffee is not expected to affect the beneficial consequences of its lipid lowering effect.

Finally, there are several factors that complicate the extrapolation of the detrimental effects of caffeine observed in experimental studies to the clinical arena. Experimental studies have examined the effect of acute [4] or short-term administration of caffeine [7]. These findings do not necessarily hold true for chronic coffee consumption, particularly because tolerance to the cardiovascular effects of caffeine has been described [13]. Also, it needs to be realized that coffee contains many compounds, other then caffeine, such as diterpene alcohols and chlorogenic acid, which can also modulate cardiovascular risk by affecting plasma cholesterol and homocysteine levels [1]. Recently, coffee consumption has been associated with a lower risk of type 2 diabetes mellitus [14], and with an increased circulating concentration of adiponectin, which has potent cardioprotective properties [15]. In epidemiological studies, these long-term beneficial effects of coffee intake may have balanced its potential deleterious effects, observed in the experimental models.

In conclusion, the association between chronic coffee consumption and cardiovascular disease is still controversial. Therefore, it is still too early to ban coffee as a measure of lifestyle modification in patients with cardiovascular disease. However, the findings from the study by Ye et al. demonstrating that coffee intake can blunt statin-induced cardioprotection [7], provides yet another experimental basis to study the acute effects of caffeine (i.e. the plasma caffeine concentration at the moment of ischaemia) on the outcome after a cardiac ischaemic event in selected patients with preinfarction ischaemia or in patients treated with statins.

Conflict of interest statement None of the authors has any conflict of interest.

Open Access This article is distributed under the terms of the Creative Commons Attribution Noncommercial License which permits any noncommercial use, distribution, and reproduction in any medium, provided the original author(s) and source are credited.

\section{References}

1. Cornelis MC, El-Sohemy A. Coffee, caffeine, and coronary heart disease. Curr Opin Lipidol 2007;18:13-9.

2. Yellon DM, Downey JM. Preconditioning the myocardium: from cellular physiology to clinical cardiology. Physiol Rev 2003; 83:1113-51.

3. Bell RM, Yellon DM. Atorvastatin, administered at the onset of reperfusion, and independent of lipid lowering, protects the myocardium by up-regulating a pro-survival pathway. J Am Coll Cardiol 2003;41:508-15.

4. Riksen NP, Zhou Z, Oyen WJ, et al. Caffeine prevents protection in two human models of ischemic preconditioning. J Am Coll Cardiol 2006;48:700-7.

5. Sanada S, Asanuma H, Minamino T, et al. Optimal windows of statin use for immediate infarct limitation: 5'-nucleotidase as another downstream molecule of phosphatidylinositol 3-kinase. Circulation 2004;110:2143-9.

6. Ye Y, Lin Y, Perez-Polo R, et al. Enhanced cardioprotection against ischemia-reperfusion injury with a dipyridamole and low- 
dose atorvastatin combination. Am J Physiol Heart Circ Physiol 2007;293:H813-818.

7. Ye Y, Abu Said G, Lin Y, et al. Caffeinated coffee blunts the myocardial protective effects of statins against ischemiareperfusion injury in the rat. Cardiovasc Drugs Ther 2008;22 (this issue) DOI 10.1007/s10557-008-6105-z.

8. Smits $P$, Thien T, van ' $t$ Laar A. The cardiovascular effects of regular and decaffeinated coffee. Br J Clin Pharmacol 1985;19:852-4.

9. Pasceri V, Patti G, Nusca A, Pristipino C, Richichi G, Di SG. Randomized trial of atorvastatin for reduction of myocardial damage during coronary intervention: results from the ARMYDA (Atorvastatin for Reduction of MYocardial Damage during Angioplasty) study. Circulation 2004;110:674-8.

10. Patti G, Pasceri V, Colonna G, et al. Atorvastatin pretreatment improves outcomes in patients with acute coronary syndromes undergoing early percutaneous coronary intervention: results of the ARMYDA-ACS randomized trial. J Am Coll Cardiol 2007;49:1272-8.
11. Cornelis MC, El-Sohemy A, Kabagambe EK, Campos H. Coffee, CYP1A2 genotype, and risk of myocardial infarction. JAMA 2006;295:1135-41.

12. Silletta MG, Marfisi R, Levantesi G, et al. Coffee consumption and risk of cardiovascular events after acute myocardial infarction: results from the GISSI (Gruppo Italiano per lo Studio della Sopravvivenza nell'Infarto miocardico)—Prevenzione trial. Circulation 2007;116:2944-51.

13. Robertson D, Wade D, Workman R, Woosley RL, Oates JA. Tolerance to the humoral and hemodynamic effects of caffeine in man. J Clin Invest 1981;67:1111-7.

14. van Dam RM, Feskens EJ. Coffee consumption and risk of type 2 diabetes mellitus. Lancet 2002;360:1477-8.

15. Williams CJ, Fargnoli JL, Hwang JJ, et al. Coffee consumption is associated with higher plasma adiponectin concentrations in women with and without type 2 diabetes: a prospective cohort study. Diabetes Care 2008;31:504-7. 